\title{
Supplementary Information: Water in Contact with a Cationic Lipid Exhibits Bulk-Like Vibrational Dynamics
}

Ruth A. Livingstone ${ }^{1 \dagger}$, Zhen Zhang ${ }^{2,3}$, Lukasz Piatkowski ${ }^{2,4}$, Huib J. Bakker ${ }^{2}$, Johannes Hunger ${ }^{1}$, Mischa Bonn ${ }^{1}$, and Ellen H.G. Backus ${ }^{1 *}$

\footnotetext{
${ }^{1}$ Max Planck Institute for Polymer Research, Ackermannweg 10, 55128 Mainz, Germany

${ }^{2}$ FOM Institute AMOLF, Science Park 104, 1098 XG Amsterdam, The Netherlands

${ }^{3}$ Chinese Academy of Sciences, 1st North St., ZhongGuanCun, HaiDian District, Beijing 100080, China

${ }^{4}$ The Institute of Photonic Sciences, Mediterranean Technology Park, 08860 Castelldefels, Spain

tCurrent Address: Center for Free Electron Laser Science, DESY, Hamburg

*Corresponding Author, backus@mpip-mainz.mpg.de
}

\section{Phase resolved Sum Frequency Generation}

The $\mathrm{H}_{2} \mathrm{O}$ - DPTAP interface was measured using phase resolved SFG spectroscopy (see ${ }^{1}$ for experimental details). The imaginary spectrum is entirely negative in the hydrogen bonding region, indicating water molecules aligned with their hydrogen atoms pointing towards the bulk. When the imaginary spectrum is scaled by -2 the spectrum can be seen to approximately overlap with the conventional SFG spectrum. This gives us confidence that measuring the tr2DSFG experiment with conventional SFG will not introduce significant artefacts.

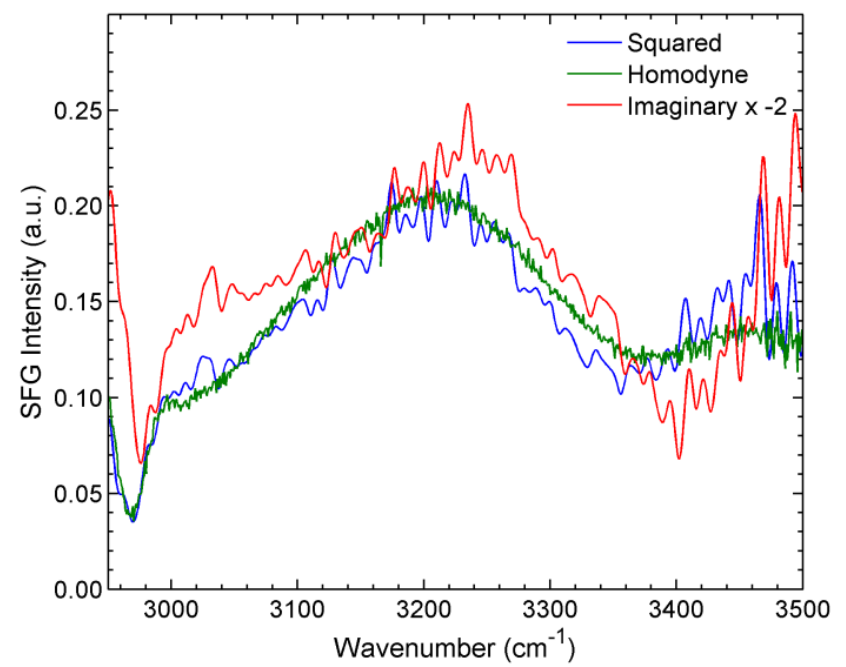

Figure S1. Phase resolved and conventional sum frequency generation spectra

\section{Four level model}

To fit the time resolved data a four level model was used. The molecules are excited from the ground state 0 to the excited state 1 with a pump pulse, whose temporal profile is estimated by a gaussian $\mathrm{G}(\mathrm{t})$. From the excited state the molecules decay to the intermediate (bend) mode $0^{*}$, with time constant $\tau_{1}$. From the intermediate state they decay to the hot ground state $0^{* *}$, with time constant $\tau_{\text {eq }}$. The following coupled differential equations describe the model:

$$
\begin{aligned}
& \frac{\mathrm{dN}_{0}}{\mathrm{dt}}=-\sigma G(\mathrm{t})\left(\mathrm{N}_{0}-\mathrm{N}_{1}\right) \\
& \frac{\mathrm{dN_{1 }}}{\mathrm{dt}}=\sigma \mathrm{G}(\mathrm{t})\left(\mathrm{N}_{0}-\mathrm{N}_{1}\right)-\frac{\mathrm{N}_{1}}{\tau_{1}} \\
& \frac{\mathrm{dN}_{0 *}}{\mathrm{dt}}=\frac{\mathrm{N}_{1}}{\tau_{1}}-\frac{\mathrm{N}_{0 *}}{\tau_{\text {eq }}} \\
& \frac{\mathrm{dN}_{0 * *}}{\mathrm{dt}}=\frac{\mathrm{N}_{0 *}}{\tau_{\text {eq }}}
\end{aligned}
$$

Where $\mathrm{N}_{\mathrm{x}}$ represents the time dependent population in state $x$, and $\sigma$ is the percentage of population that is excited by the pump pulse. The populations are then used to calculate the expected change in SFG signal as a function of pump-probe time delay as follows:

$$
\Delta \mathrm{SFG}(\mathrm{t})=\mathrm{S}\left(\left(\left(\mathrm{N}_{0}+\mathrm{N}_{0 *}-\mathrm{N}_{1}\right)+\mathrm{O}_{\mathrm{hot}} \mathrm{N}_{0 * *}\right)^{2}-1^{2}\right)
$$

$S$ is a scaling factor which is related to the SFG signal intensity. $\mathrm{O}_{\text {hot }}$ is the magnitude of the offset due to thermal effects from the hot ground state, measured at long time delays.

In all the fits $\tau_{\text {eq }}$ was fixed to $700 \mathrm{fs}, \mathrm{O}_{\text {hot }}$ was fixed from the measurements. To fit the data $S$ and $\tau_{1}$ were varied. The individual fits for pure $\mathrm{D}_{2} \mathrm{O}$ and $\mathrm{H}_{2} \mathrm{O}: \mathrm{HOD}: \mathrm{D}_{2} \mathrm{O}=1: 2: 1$ are shown in figure $S 2$ and figure $5(a)$. The $\tau_{1}$ extracted from these fits can be seen in figure $5(b)$. 


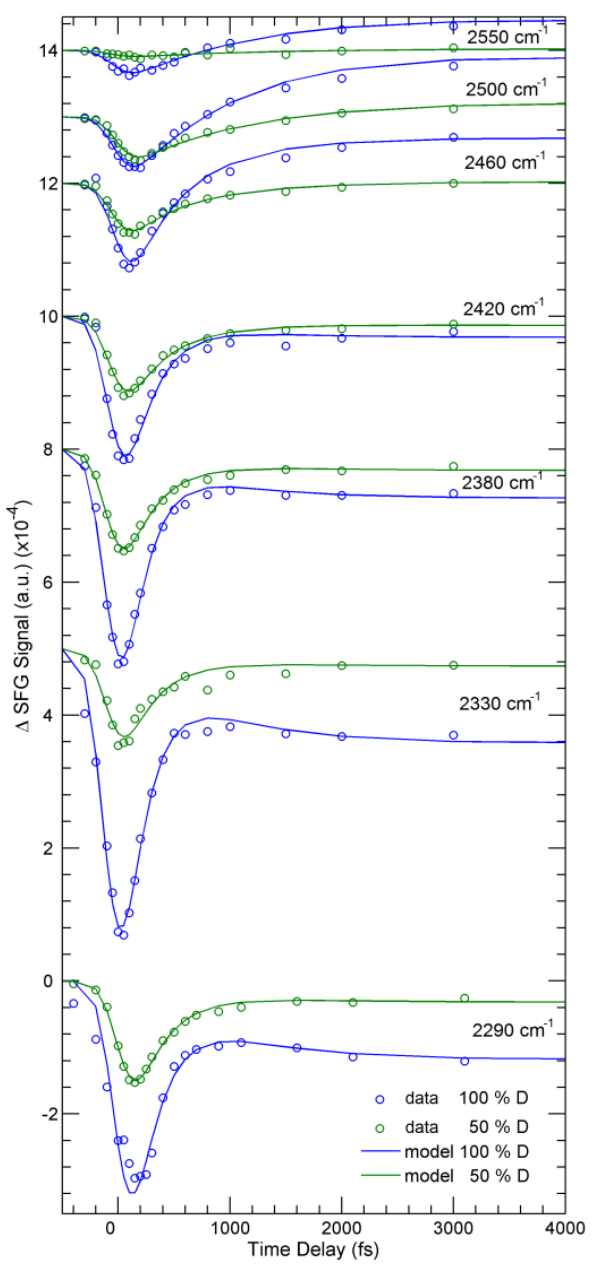

Figure S2: Time resolved data (circles) for two different isotopic dilutions and fits (lines) using coupled differential equations outlined in the text. The data for the different pump frequencies are offset for clarity (pump frequencies are annotated). The data points are the differential SFG signal averaged over $50 \mathrm{~cm}^{-1}$ centered at the pump frequency for each pump-probe time delay.

\section{Two dimensional Spectra}

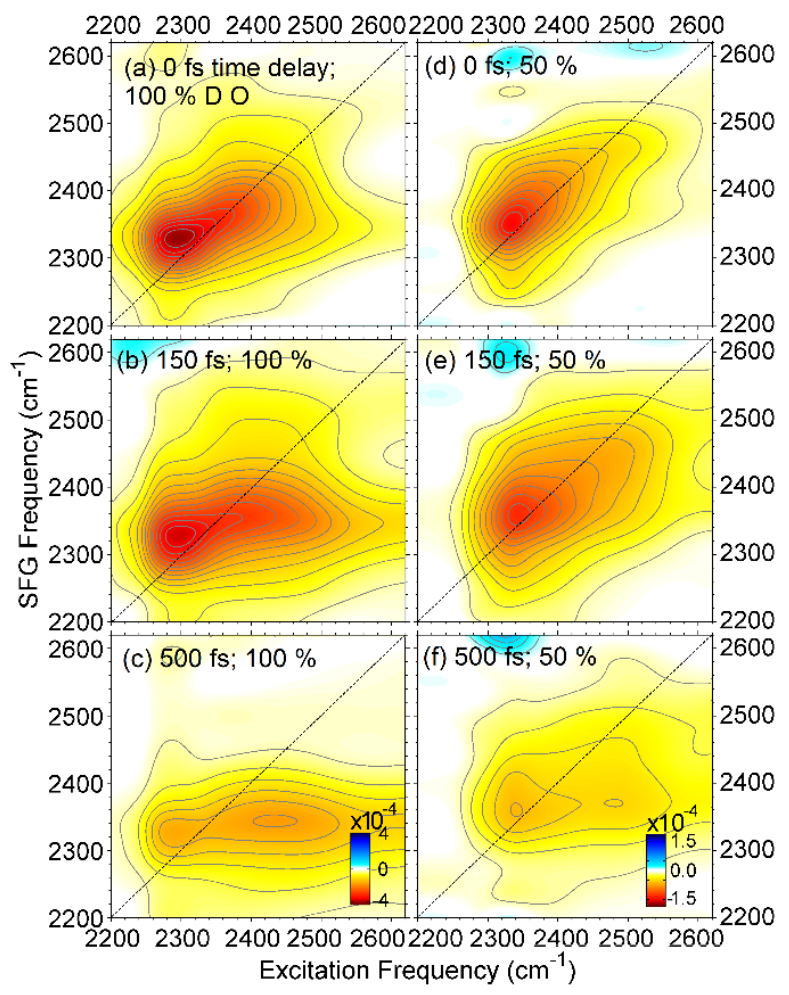

Figure S3: 2D spectra without heat subtraction

\section{References}

1. Pool, R. E.; Versluis, J.; Backus, E. H. G.; Bonn, M., Comparative Study of Direct and Phase-Specific Vibrational Sum-Frequency Generation Spectroscopy: Advantages and Limitations. The Journal of Physical Chemistry B 2011, 115 (51), 15362-15369. 